\title{
Sequence and Equivalent of Reagents Addition in NHC-Catalyzed Oxidative Nonpolar Inversion Enable Conversion from Aldimine to Benzoxazole
}

\author{
Xiaoyan $\mathrm{Li}^{1}$, Jiabin Liu ${ }^{1}$, Qiao-Chu Zhang ${ }^{1}$, Wenjing Zhang ${ }^{1}$, and Yu Lan ${ }^{2}$ \\ ${ }^{1}$ Zhengzhou University \\ ${ }^{2}$ Chongqing University
}

May 5, 2020

\begin{abstract}
Mechanism of the oxidative nonpolar inversion reaction catalyzed by N-heterocyclic carbenes (NHCs) to achieve benzoxazoles was investigated in very details. The reaction was revealed to occur through five processes, and for oxidation in the second process, two successive tautomerizations followed by oxidation were demonstrated to be more energetically favorable than the other two pathways. The rate-determining step was disclosed to be the oxidation by 3,3'-5,5'-tetra-tert-butyl-4,4'-diphenoquinone (DQ). Afterwards, mechanism calculations to the non-catalyzed reaction was conducted and it was revealed that the excessive exothermic property of the initial step should be the main reason for the extremely high barrier in the following step. While with participation of NHC, this unfavorable transformation can be deftly prevented according to the specific sequence and amount of reagents addition, and therefore to enable the reaction to occur under mild conditions.
\end{abstract}

Sequence and Equivalent of Reagents Addition in NHC-Catalyzed Oxidative Nonpolar Inversion Enable Conversion from Aldimine to Benzoxazole

Xiaoyan Li, 11College of Chemistry, and Institute of Green Catalysis, Zhengzhou University, Zhengzhou, Henan Province 450001, P. R. China Jiabin Liu, ${ }^{1}$ Qiao-Chu Zhang, ${ }^{1}$ Wenjing Zhang, ${ }^{1}$ and Yu Lan ${ }^{1}$

Correspondence to: Wenjing Zhang and Yu Lan (E-mail:zhangwj@zzu.edu.cn,lanyu@cqu.edu.cn)

\section{Introduction}

Since the crystalline carbene 1,3-di-l-adamantyl-imidazol-2-ylidene was firstly isolated and characterized by Arduengo et al., ${ }^{1,2}$ extensive researches emerge involving synthesis of more stable N-heterocyclic carbenes (NHCs) and applications of $\mathrm{NHC}$ as ligand of metal-based catalyst or organocatalyst. ${ }^{3}$ Particularly, the success in catalytic benzoin condensations promotes the NHC catalysis as an important alternative in constructing new $\mathrm{C}-\mathrm{C}$ bonds and $\mathrm{C}-\mathrm{X}(\mathrm{X}=\mathrm{N}, \mathrm{O}, \mathrm{S})$ bonds. ${ }^{4-9}$ Inspired by the 'umpolung' principle used in this reaction, ${ }^{10,11}$ the NHC-catalyzed Stetter reaction, ${ }^{12}$ benzoin reaction, ${ }^{13}$ homoenolate reaction, and especially cycloaddition/annulation reactions ${ }^{14-18}$ have contributed a number of novel approaches to constructing various heterocyclic structures. Inversion of the conventional reactivity of substrates, typically such as aldehyde, ketone, ketene, and so on, is the basic underline principle behind these reactions (Scheme 1). ${ }^{19-21}$ More interestingly, catalysis using this concept has been extended to the umpolung of Michael acceptors $^{22-25}$ and aldimines. ${ }^{26-33}$ Detailed theoretical studies reported by our ${ }^{34-42}$ and other groups ${ }^{43-51}$ provide perspective at the molecular level to get insights into the catalytic mechanism and chemo-, regio-, and/or stereoselectivities. ${ }^{52,53}$ 


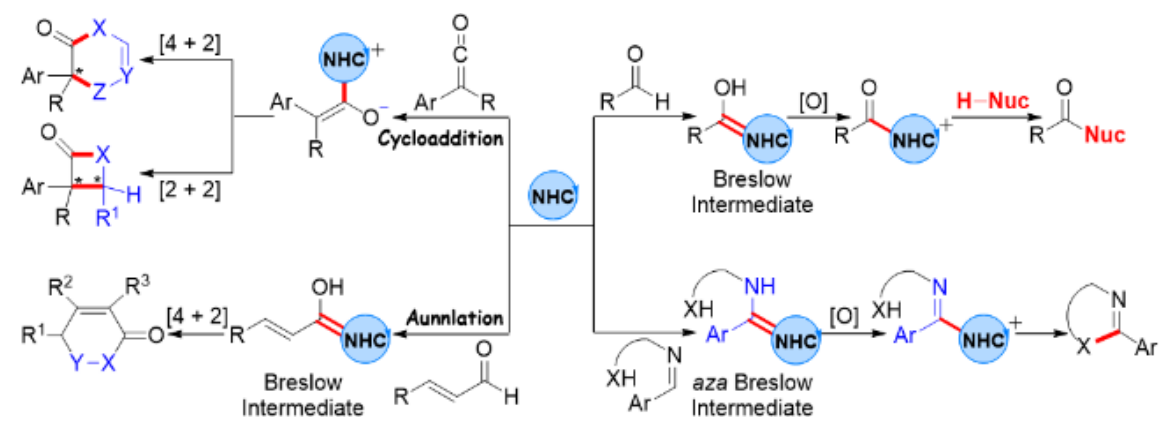

Scheme 1. Selected NHC-catalyzed reactions based on the umpolung strategy (left) or the oxidative nonpolar inversion strategy (right).

The fact is 'umpolung' should not be the unique approach for NHCs as organocatalyst. ${ }^{54-57}$ As it is illustrated in Scheme 1, in the normal way without polarity inversion under oxidative conditions, the reaction can be initiated by conversion of aldehyde or aldimine to the Breslow or aza-Breslow intermediate ${ }^{58}$ respectively, followed by a two electron oxidation to give azolium. Finally, an inter- or intramolecular nucleophilic attack yields the final product. For these reactions, the roles of NHCs play in facilitating reactions to occur and/or manipulation of various selectivities is an important issue that people are highly interested in. In the 'umpolung' reactions, the main effect of NHCs has been demonstrated to be increasing nucleophilicity of the substrate or determining the selectivities owing to diverse non-covalent interactions (NCIs) between substituents of the catalyst and the substrate in different isomers. While for the nonpolar inversion pathway, acts of the catalysts could be complicated, such as enhancing reducibility of the substrate or even shows completely a diverse reaction route. Owing to our long interesting in the NHC-catalyzed reactions but the lack of insights into the roles of $\mathrm{NHC}$ and the co-effect of the oxidant in reactions without polarity reverse, we would like to present our recent theoretical study about the mechanism of an intramolecular oxidative cyclization with $\mathrm{NHC}$ as the catalyst and 3,3'-5,5'-tetra-tert-butyl-4,4'-diphenoquinone (DQ) ${ }^{59}$ as the oxidation reagent. More importantly, some insights about how the catalyst and DQ work together to enable the reaction have also been achieved.

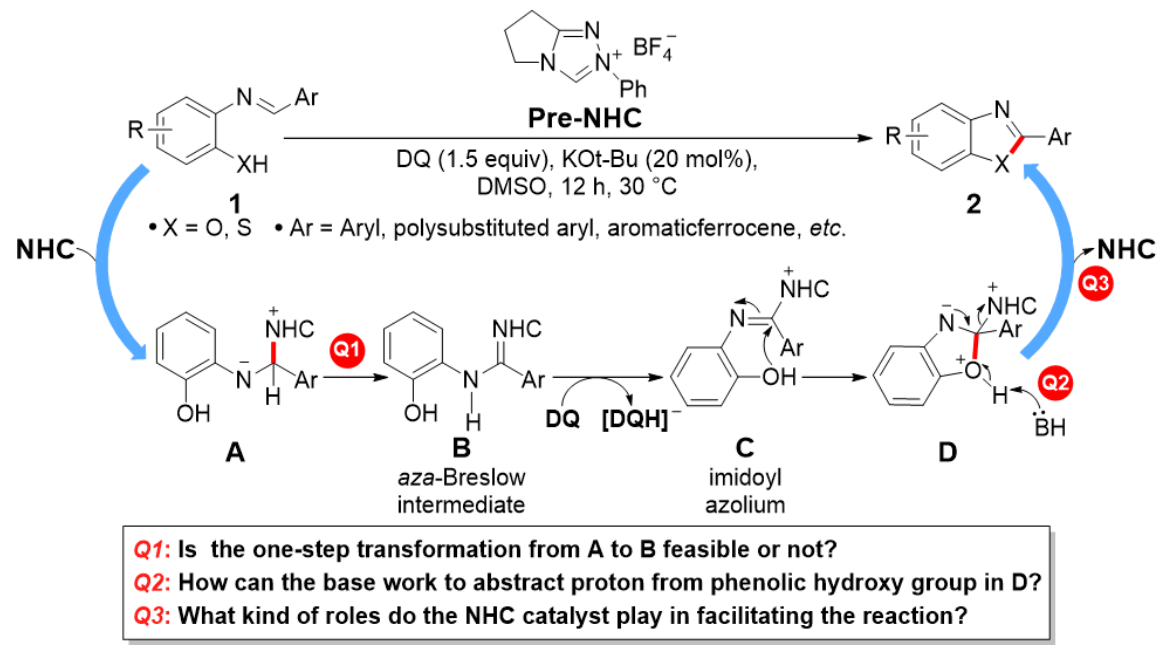

Scheme 2. Experimental details and the proposed tentative mechanism.

More details about the experimental results by Biju and co-workers ${ }^{60}$ were shown in Scheme 2. The aldimine 1 was treated with the carbene that is generated in situ from triazolium salt Pre-NHC using $\mathrm{KO} t-\mathrm{Bu}$ as 
the base and DQ as the oxidant. The optimal solvent was demonstrated to be DMSO, and the temperature was set at $30{ }^{\circ} \mathrm{C}$. Various 2-arylbenzoxazole and 2-arylbenzothiazole were obtained with yield up to $99 \%$. The tentative mechanism indicates five steps for the whole catalytic cycle, namely absorption of NHC to the substrate to give zwitterion A, followed by [1,2]-proton transfer to generate the aza-Breslow intermediate $\mathrm{B}$, oxidation by $\mathrm{DQ}$, ring closure under assistance of the Lewis base, and finally desorption of the catalyst. This cycle provides inspired perspectives to get better understanding to the general process of the catalytic mechanism, but its rationality and feasibility still need to be verified. For example: since the [1,2]-proton transfer usually difficult, ${ }^{61}$ is the one-step transformation from intermediate A to aza-Breslow intermediate $\mathrm{B}$ feasible or not? Since the triazolium salt Pre-NHC and the base $\mathrm{KO} t$-Bu were added in exactly equal equivalent, how it can be used in proton abstraction from phenolic hydroxy group in compound D? What kind of roles do the NHC catalyst play in enabling the reaction to occur? In order to pursue deep insights into all above questions, we conducted detailed density functional theory (DFT) ${ }^{62}$ studies to the entire catalytic cycle.

\section{Computational Details}

All theoretical calculations were carried out by using theGaussian09 suit of program. ${ }^{63}$ Structures of all stationary points, including the reactants, products, transition states and intermediates, were fully optimized by using the M06-2X functional ${ }^{64-66}$ and the $6-31 \mathrm{G}(\mathrm{d}, \mathrm{p})$ basis set ${ }^{67,68}$ under gas phase (Level $A$ ). The harmonic frequency calculations were performed at the same level with the temperature set as $303.15 \mathrm{~K}$ and the pressure as $1 \mathrm{~atm}$ according to the experimental report. All reactants, products, and intermediates were corroborated to have no imaginary frequency while each transition state to have one and only one imaginary frequency. The same level of intrinsic reaction coordinate calculations (IRC) ${ }^{69,70}$ were carried out to confirm each transition state linked the expected reactant and product. Based on the optimized structures, all energies were refined by single-point calculations at the M06-2X/6-311++G(d, p $)^{71}$ level of theory, with the solvent effects of DMSO simulated by Truhlar's SMD ${ }^{72,73}$ model (Level $B$ ). All energies discussed in this paper were calculated by adding the single-point energies obtained at Level $B$ to the thermal corrections of the Gibbs free energies calculated atLevel $A$. 


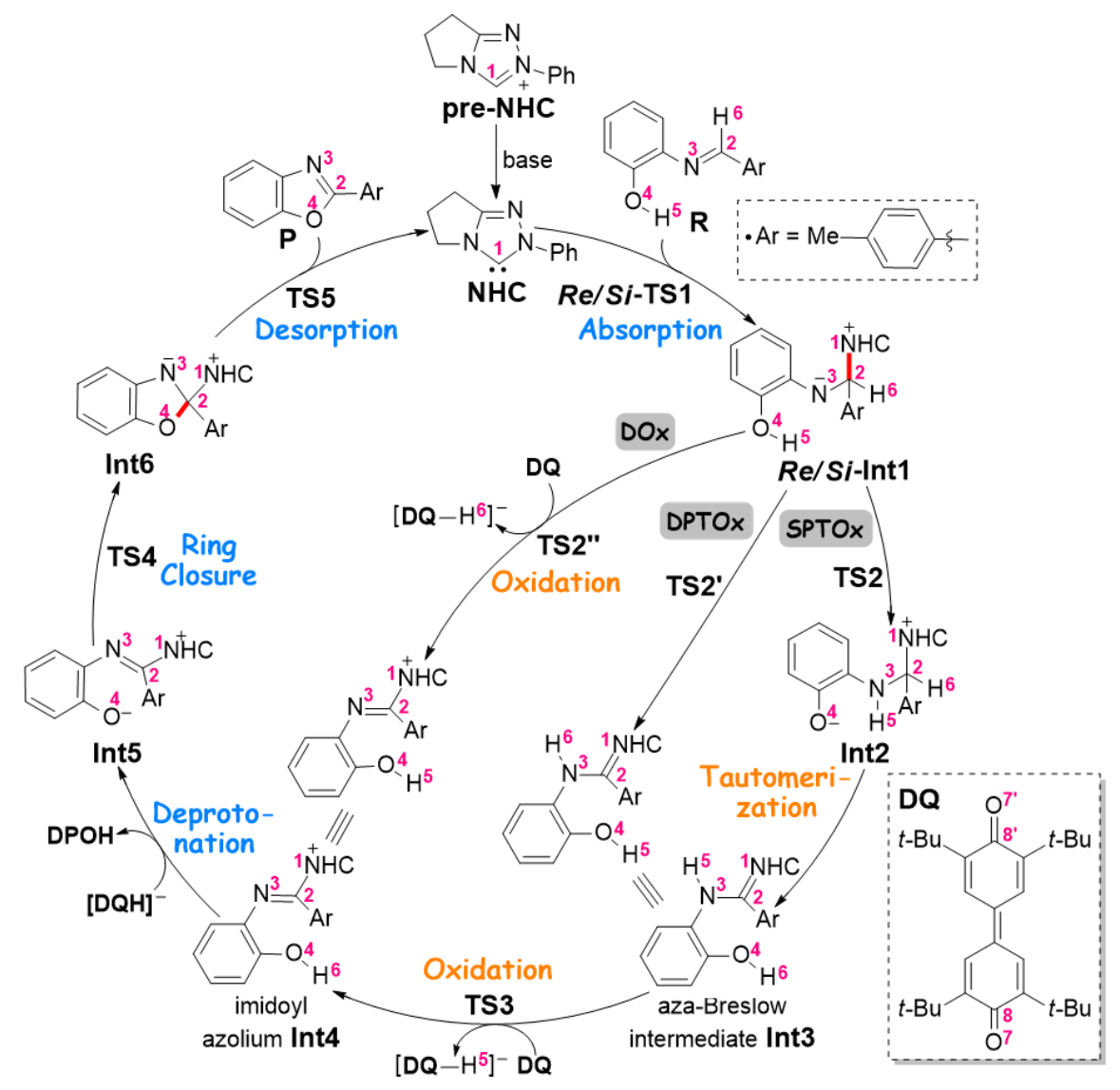

Scheme 3. The proposed mechanism of the title reaction.

\section{Results and Discussion}

\subsection{Reaction Mechanism}

The catalytic transformation of ( $E$ )-2-((4-methylbenzylidene)amino)phenol (denoted as $\mathbf{R}$ ) was selected as the computational model because it gives 4-tolylbenzoxazole with excellent yield of $99 \%$. We present the entire catalytic cycle in Scheme 3 with more details considered, especially for the tautomerization from zwitterion intermediate Int1, which is generated by absorption of the NHC catalyst (denoted as Cat ) to the aldimine $\mathbf{R}$, to the imidoyl azolium Int4 . There are three possible pathways calculated and more discussions will be given below. The following processes are successively deprotonation from the phenolic hydroxy group under assistance of [DQH $]^{-}$(4'-hydroxy-[1,1'-biphenyl]-4-olate), ring-closure, and finally desorption of the product $\mathbf{P}$ from Cat . In the following part of this section, we will give detailed discussions about this catalytic cycle step by step. The Gibbs free energy profiles of the whole reaction along with some key geometry parameters of all transition states are presented in Figure 1. Unless otherwise stated, the energies of $\mathbf{C a t}+\mathbf{R}$ were set as the reference of $0.0 \mathrm{kcal} / \mathrm{mol}$.

In the first process, the nucleophilic attack of the $\mathrm{C} 1$ atom inCat to the $\mathrm{C} 2$ atom in $\mathbf{R}$ may occur from either the Re- or $S i$ - face. The resulting transition stateRe-TS1 was predicted to locate $3.1 \mathrm{kcal} / \mathrm{mol}$ higher thanSi-TS1, and the corresponding absorption product $S i$-Int1 was found to be $8.3 \mathrm{kcal} / \mathrm{mol}$ more stable than that Re-Int1. Thus we abandoned all the following calculations related with Re-Int1 . Besides, the barrier via Si-TS1 was calculated to be $20.3 \mathrm{kcal} / \mathrm{mol}$, indicating that the absorption is able to occur smoothly under the experimental conditions $\left(30^{\circ} \mathrm{C}\right)$. 
Given the zwitterion intermediate $S i$-Int1, three possible pathways for its oxidation to generate the imidoyl azoliumInt4 were calculated, in particular the direct oxidation byDQ via transition state TS2" (DOx), the direct [1,2]-proton transfer of the H6 atom via TS2' to give aza-Breslow intermediate Int3 and then followed by oxidation byDQ to form Int4 (DPTOx), and the successive transformations of [1,4]-proton transfer of the H5 atom viaTS2 /spontaneous [1,5]-proton transfer of the H6 atom/oxidation by DQ via TS3 to form Int4(SPTOx).

In the DOx pathway, the hydride $\mathrm{H} 6$ was abstracted from the $\mathrm{C} 2$ atom inSi-Int1 to the O7 atom in DQ . As shown by the optimized geometry parameters in Figure S1, the length of the $\mathrm{C} 2-\mathrm{H} 6$ bond is elongated from $1.11 \AA$ in $S i$-Int1 to $1.23 \AA$ inTS2", while distance between the $\mathrm{H} 6$ and $\mathrm{O} 7$ atoms is shortened from $1.27 \AA$ in TS2" to $0.96 \AA$ in $[\mathrm{DQH}]^{-}$, which clearly indicates formation of the new bond. The barrier of this elementary step was predicted to be $41.0 \mathrm{kcal} / \mathrm{mol}$ (Figure 1), which is too high to be overcome under the experimental conditions. Consequently, the reaction goes through the DOx strategy was excluded.

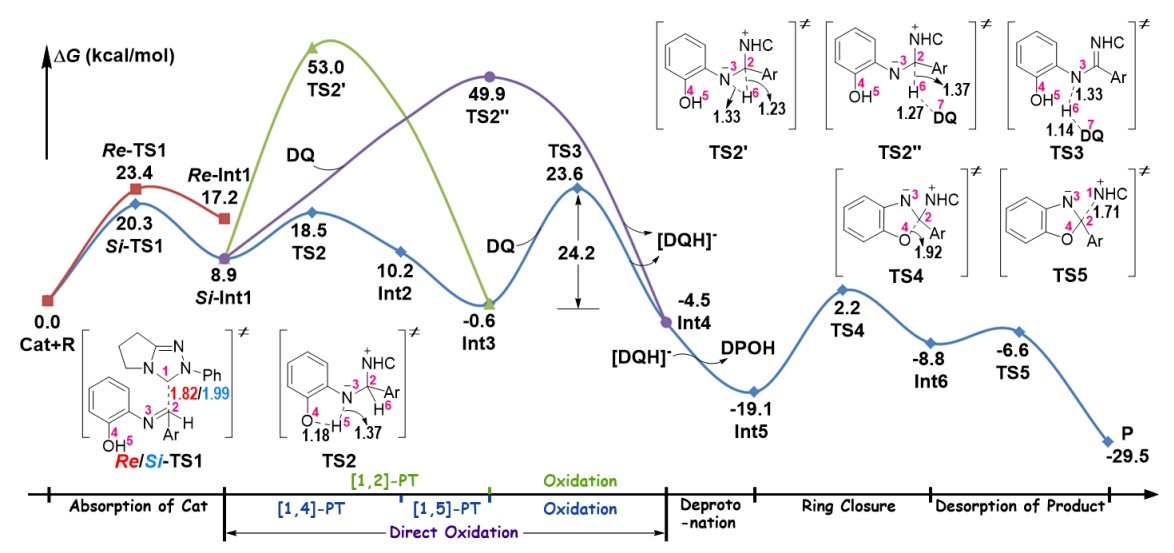

Figure 1. Gibbs free energy profiles of the whole reaction and some key geometry parameters of all transition states involved. All bond lengths are given in angstrom $(\AA)$.

The DPTOx pathway assumes a typical [1,2]-proton transfer of the $\mathrm{H} 6$ atom from the $\mathrm{C} 2$ atom to the N3 atom so as to provide the aza-Breslow intermediate Int3, which has been definitely verified to be difficult under mild conditions due to the highly strained three-membered ring involved in the transition state $(\mathrm{C} 2-$ N3-H6 inTS2' ). Not surprisingly, the Gibbs free energy barrier viaTS2' was predicted to be $44.1 \mathrm{kcal} / \mathrm{mol}$, which is obviously an unreasonable obstacle for the mild experimental conditions.

In the SPTOx pathway, two successive proton transfers were proposed to give Int3, in particular the proton H5 shifted from the $\mathrm{O} 4$ atom to the negative N3 atom via transition state TS2, followed by the migration of the $\mathrm{H} 6$ atom from the $\mathrm{C} 2$ atom back to the $\mathrm{O} 4$ atom to generate intermediate Int3. The energy barrier via TS2 was calculated to be $9.6 \mathrm{kcal} / \mathrm{mol}$, and the tautomerization fromInt2 to Int3 was demonstrated to be spontaneous via the flexible scanning of the $\mathrm{O} 4-\mathrm{H} 6$ bond length (Please see Figure $\mathrm{S} 2$ for more details). The subsequent elementary step is to oxidateInt3 to Int4 via hydride transfer of the H5 atom from the N3 atom to DQ . The gradual elongation of the N3-H5 bond length (from 1.02 $\AA$ in Int3 to $1.33 \AA$ in TS3, Figure S1) and the continued shortening of the O7-H5 bond length (from $1.14 \AA$ in TS3 to $0.96 \AA$ in $[\mathbf{D Q H}]^{-}$ , Figure S1) clearly indicated the abstraction of the hydride H5 byDQ . As shown in Figure 1, transition state TS3 was predicted to locate $24.2 \mathrm{kcal} / \mathrm{mol}$ higher than the intermediateInt3, which is much lower than that goes through the DOx or DPTOx pathway. Taking all these discussions into consideration, the SPTOx pathway was concluded to be most energetically favorable for oxidation from Si-Int1 to the imidoyl azoliumInt4 .

The third process is to abstract the $\mathrm{H} 6$ atom from the phenolic hydroxy group by the [DQH $]^{-}$to afford the zwitterion intermediate Int5 . It was confirmed to be also barrierless by the flexible scanning of the O4- 
H6 bond length (Please see Figure S3 for more details). The fourth process is the ring closure undergoing through nucleophilic attack of the $\mathrm{O} 4$ atom to the $\mathrm{C} 2$ atom to form intermediate Int6 via transition state TS4 . The geometry optimizations indicates that the distance between the $\mathrm{C} 2$ and $\mathrm{O} 4$ atoms decreases from $2.39 \AA$ in Int5 to $1.92 \AA$ in TS4and finally become $1.44 \AA$ in Int6, which clearly demonstrates formation of the $\mathrm{C} 2-\mathrm{O} 4$ bond. The energy barrier of this step was predicted to be $21.3 \mathrm{kcal} / \mathrm{mol}$, indicating that this intramolecular cyclization process can be accomplished under the experimental conditions. Finally, formation of the $\mathrm{C} 2=\mathrm{N} 3$ double bond promoted release of the final product $\mathbf{P}$ from the catalyst. The very low energy barrier via transition state TS5 $(2.2 \mathrm{kcal} / \mathrm{mol}$, shown in Figure 1) indicates that it is easy for the catalyst to be recycled, which corroborates the potential for the NHC as a good leaving group.

\subsection{Roles of NHC}

As an organocatalyst, the NHC species work generally to enhance electrophilicity of the substrate, to manipulate stereoselectivities by rational regulations of the non-covalent interactions between substituents of the chiral catalyst and the substrate, or most importantly to inverse polarity of the ketene, ketone, or aldehyde to expand applications of NHCs in synthesis of heterocyclic compounds. In regarding the title reaction we are interested in, the experimental results from Biju and co-workers indicate that the yield can be dramatically decreased to be $7 \%$ when all reagents were set under exactly the same conditions but with NHC absent and the phenyl substituted aldimine replaced by benzyl substituted aldimine. Moreover, according to all the above discussions about the catalytic cycle, it is easy to conclude that the reaction undergoes without polarity reversal. So what kind of roles do the NHC catalyst act in this transformation from o-amino phenol to benzoxazole? Why it is so important for promoting the transformation going efficiently?

In order to get some insights into these queries, we then conducted mechanism studies to the reaction without participation of NHC. As illustrated in Figure 2(a), the whole reaction was proposed to occur through four steps, namely deprotonation of the phenolic hydroxy group in $\mathbf{R}_{\mathbf{n c}}$ to give ionic compoundInt $\mathbf{1}_{\mathbf{n c}}$, followed by a conformation isomerism of the aldimine group to give $\mathbf{I n t} \mathbf{2}_{\mathbf{n c}}$. Subsequently, two possible transformation pathways were considered, i.e. ring closure through nucleophilic attack of the $\mathrm{O} 4$ atom to the $\mathrm{C} 2$ atom followed by oxidation by $\mathbf{D Q}$, or the oxidation of the $\mathrm{C} 2$ atom goes first, followed by ring closure through bonding of the $\mathrm{C} 2$ and $\mathrm{O} 4$ atoms. The predicted Gibbs free energy profiles of the whole reaction were presented in Figure $2(\mathrm{~b})$, and the energies of $\mathbf{R}_{\mathbf{n c}}+\mathrm{KO} t-\mathrm{Bu}$ was set as the reference of $0.0 \mathrm{kcal} / \mathrm{mol}$ unless otherwise specified. 

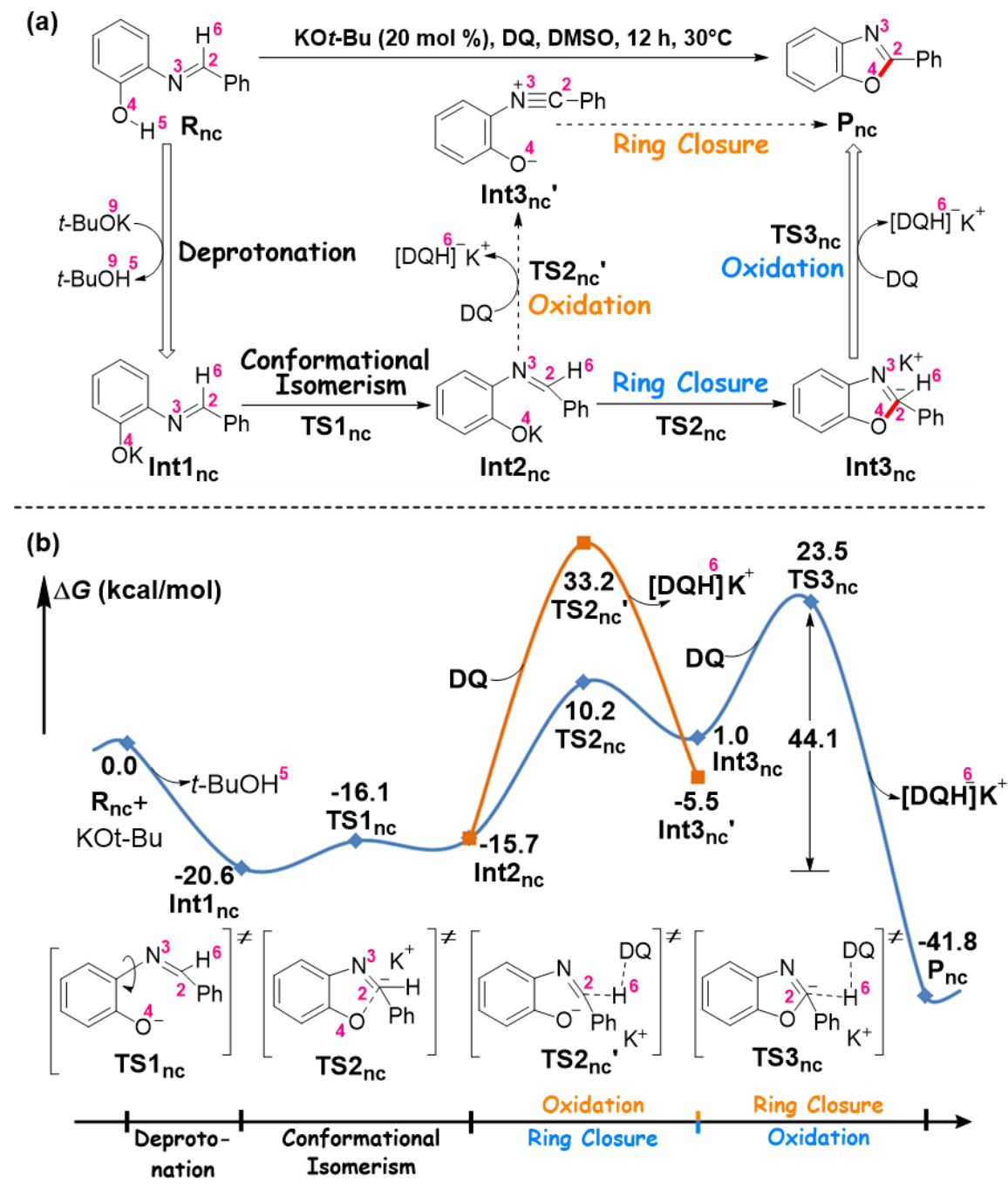

Figure 2. (a) The proposed mechanism for the reaction without NHC catalysis, and (b) the predicted Gibbs free energy profiles along with some key parameters in each transition states (bond length in $\AA$ ).

The computational results reveal that the initial step of the reaction is barrierless (See Figure S4 for more details) and remarkably thermodynamically favorable, indicating strong preference of the proton transfer from phenolic hydroxy group to the tert-butyl alcohol anion $\left(t-\mathrm{BuO}^{-}\right)$. The energy barrier for the conformational transformation via $\mathbf{T S} \mathbf{1}_{\mathbf{n c}}$ was predicted to be $4.5 \mathrm{kcal} / \mathrm{mol}$, which is very easy to be overcome under mild conditions. However, the following conversion fromInt $\mathbf{2}_{\mathbf{n c}}$ to the benzoxazole product $\mathbf{P}_{\mathbf{n c}}$ was demonstrated to be very difficult via neither of the two proposed pathways because of their extremely high barrier (44.1 or $53.8 \mathrm{kcal} / \mathrm{mol}$, respectively).

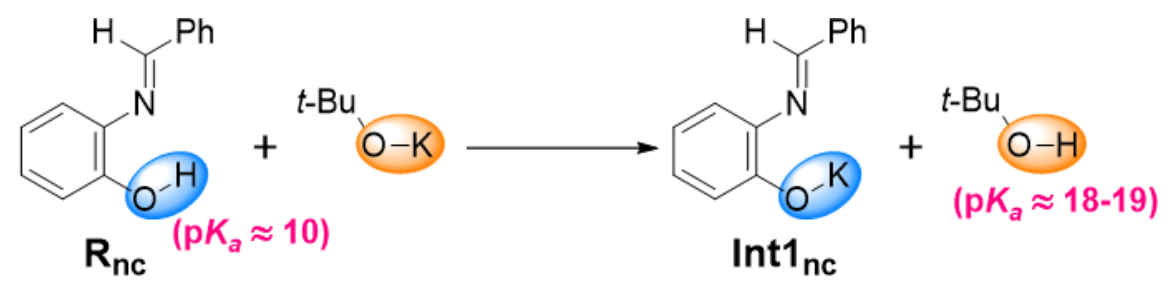


Scheme 4. The main change in the initial step of the non-catalyzed reaction.

Taking all above discussions into consideration, for either the NHC-catalyzed or non-catalyzed reaction, the oxidation by DQwas disclosed to be the rate-determining step (RDS), but the barrier of the latter strategy $(44.1 \mathrm{kcal} / \mathrm{mol})$ is about $20 \mathrm{kcal} / \mathrm{mol}$ higher than the former one $(24.2 \mathrm{kcal} / \mathrm{mol})$. Actually, due to participation of NHC, the reaction mechanism has been fundamentally altered, leading to the whole barrier changes from the accumulate obstacles including the conformational isomerism, the ring closure and the oxidation processes to the barrier of the elementary oxidation step. In addition, the energies of the highest point in the two energy profiles (TS3and $\mathbf{T S 3}_{\mathbf{n c}}$, respectively) are almost equal $(23.6 \mathrm{vs} 23.5 \mathrm{kcal} / \mathrm{mol})$, indicating that it should be the excessive exothermic change in the initial deprotonation step that leads to the unreasonable barrier of the non-catalyzed reaction. As illustrated in Scheme 4, it is no surprise that the latter state locates more than $20 \mathrm{kcal} / \mathrm{mol}$ lower than the initial state because this is typically a displacement reaction of strong acid with strong base to give relatively weak acid and weak base. While with participation of NHC, this transformation is subtly prevented by controlling the reaction procedure. In particular, exactly equal amount $(20 \mathrm{~mol} \%$ ) of triazolium salt Pre-NHC and the base $t$-BuOK were firstly stirred in the DMSO solvent for $15 \mathrm{~min}$ to yield the NHC catalyst in situ. Afterwards, there was littlet -BuOK left to react with the substrate which was later added to the system along with the oxidant. In a word, by properly controlling the order and amount of reagents added, the reaction mechanism is completely changed by use of NHC catalyst. This strategy effectively prevents formation of the excessive stable intermediate, and thus resulting in substantial reduction in energy barrier of the whole reaction.

\section{Conclusions}

The mechanism of the oxidative nonpolar inversion reaction catalyzed by NHC to achieve benzoxazoles and roles of the NHC catalysis in facilitating the reaction were investigated in very details. It was revealed that the reaction goes through generally five processes, i.e. absorption of the catalyst, oxidation of the zwitterion intermediate to imidoyl azolium, deprotonation followed by ring closure, and finally desorption of the catalyst. As for the oxidation process, the successive [1,4]-/[1,5]-proton transfers followed by oxidation byDQ was demonstrated to be much more energetically favorable than the direct oxidation or the direct proton transfer followed by oxidation pathway. Oxidation of the $\mathrm{C} 2$ atom was indicated to be the RDS, and the barrier was predicted to be $24.2 \mathrm{kcal} / \mathrm{mol}$, a reasonable value for experimental conditions $\left(30{ }^{\circ} \mathrm{C}\right)$. Mechanism of the non-catalyzed reaction was also calculated, and the excessive exothermic property of the initial step was concluded to be the main reason for its extremely high barrier. In the NHC-catalyzed reaction, this unfavorable transformation was subtly prevented due to the specific sequence and amount of reagents addition. We conclude that the intrinsic requirement of NHC catalyst formation in situ and the exactly equal amount of the Pre-NHC and $t$-BuOK are the two core factors that enable the reaction to occur under mild conditions.

\section{Funding Information}

The work described in this study was supported by the National Natural Science Foundation of China (No. 21503191) and the China Postdoctoral Science Foundation (No. 2015M572115).

Keywords: NHC Catalysis; Reaction Mechanism and Roles of Catalyst; Density Functional Theory; Nonpolar Inversion; Oxidation

Additional Supporting Information may be found in the online version of this article.

\section{References}

1. A. J. Arduengo, R. L. Harlow, M. Kline, J. Am. Chem. Soc. 1991 , 113 , 361-363.

2. J. C. Garrison, W. J. Youngs, Chem. Rev. 2005 , 105 , 3978-4008.

3. E. Peris, R. H. Crabtree,Coord. Chem. Rev. 2004, 248, 2239-2246.

4. K. J. R. Murauski, A. A. Jaworski, K. A. Scheidt, Chem. Soc. Rev. 2018, 47 , 1773-1782. 
5. D. M. Flanigan, F. Romanov-Michailidis, N. A. White, T. Rovis, Chem. Rev.2015 , 115 , 9307-9387.

6. R. S. Menon, A. T. Biju, V. Nair,Chem. Soc. Rev. 2015 , 44 , 5040-5052.

7. M. N. Hopkinson, C. Richter, M. Schedler, F. Glorius, Nature 2014, 510 , 485-496.

8. A. T. Biju, N. Kuhl, F. Glorius, Acc. Chem. Res. 2011, 44 , 1182-1195.

9. D. Enders, O. Niemeier, A. Henseler, Chem. Rev. 2007 , 107, 5606-5655.

10. F. Wöhler, J. Liebig, Ann. Der. Pharm. 1832 , 3 , 249-282.

11. D. Seebach, Angew. Chem., Int. Ed. Engl. 1979 , 18 , 239-336.

12. H. Stetter, Angew. Chem. Int. Ed. Engl. 1976, $15,639-712$.

13. J. U. Nef, Justus Liebigs Ann. Chem. 1895, 287, 265-359.

14. Y. Wang, D. Wei, W. Zhang, ChemCatChem. 2018, 10 , 338-360.

15. X. Bugaut, F. Liu, F. Glorius, J. Am. Chem. Soc. 2011, 133 , 8130-8133.

16. D. T. Cohen, B. Cardinal-David, J. M. Roberts, A. A. Sarjeant, K. A. Scheidt, Org. Lett.2011, 13 , 1068-1071.

17. M.-Q. Jia, S.-L. You, ACS Catal. 2013 , 3 , 622-624.

18. X. Zhao, D. A. DiRocco, T. Rovis, J. Am. Chem. Soc. 2011 , 133 , 12466-12469.

19. D. Wei, Y. Zhu, W. Zhang, M. Tang, J. Mol. Catal. A: Chem. 2011 , 334 , 108-115.

20. D. Wei, Y. Wang, Org. Biomol. Chem. 2014, 12, 6374-6383.

21. X. Zhang, M. Tang, Y. Wang, Y. Ran, D. Wei, Y. Zhu, W. Zhang, J. Org. Chem. 2016 ,81 , 868-877.

22. Y. Nakano, D. W. Lupton,Angew. Chem. Int. Ed. 2016 , 55 , 3135-3139.

23. S.-i. Matsuoka, Y. Ota, A. Washio, A. Katada, K. Ichioka, K. Takagi, M. Suzuki, Org. Lett.2011, 13 , $3722-3725$.

24. A. T. Biju, M. Padmanaban, N. E. Wurz, Angew. Chem. Int. Ed. 2011 , 50 , 8412-8415.

25. C. Fischer, S. W. Smith, D. A. Powell, G. C. Fu, J. Am. Chem. Soc. 2006 , 128, 1472-1473.

26. A. Patra, F. Gelat, X. Pannecoucke, T. Poisson, T. Besset, A. T. Biju, Org. Lett.2018, 20 , 1086-1089.

27. A. Patra, S. Mukherjee, T. K. Das, S. Jain, R. G. Gonnade, A. T. Biju, Angew. Chem. Int. Ed.2017, $56,2730-3734$.

28. B. Harish, M. Subbireddy, S. Suresh, Chem. Commun. 2017, 53 , 3338-3341.

29. S. J. Lee, H.-A. Seo, C.-H. Cheon, Adv. Synth. Catal. 2016 , 358 , 1566-1570.

30. T. K. Das, S. Mondal, R. G. Gonnade, A. T. Biju, Org. Lett. 2017 , 19 , 5597-5600.

31. Biplab Maji, Markus Horn, H. Mayr, Angew. Chem. Int. Ed. 2012 , 51, 6231-6235.

32. C. E. I. Knappke, J. M. Neudörfl, A. Jacobi von Wangelin, Org. Biomol. Chem. 2010 ,8 , 1695-1705.

33. C. E. I. Knappke, A. J. Arduengo III, H. Jiao, J. M. Neudörfl, A. Jacobi von Wangelin, Synthesis2011 , $23,3784-3795$.

34. Y. Y. Ran, M. S. Tang, Y. Wang, Y. Y. Wang, X. L. Zhang, Y. Y. Zhu, D. H. Wei, W. Zhang, Tetrahedron $2016,72,5295-5300$.

35. Y. Wang, B. Wu, L. Zheng, D. Wei, M. Tang, Org. Chem. Front. 2016 , 3 , 190-203. 
36. X. Guo, L. B. Zhang, D. H. W. and, J. L. Niu, Chem. Sci. 2015 , 6 , 7059-7071.

37. Y. Wang, D. Wei, Z. Li, Y. Zhu, M. Tang, J. Phys. Chem. A 2014, 118 , 4288-4300.

38. A. F. Littke, G. C. Fu,Angew. Chem. Int. Ed. 2002 , 41, 4176-4211.

39. W. Zhang, Y. Wang, D. Wei, M. Tang, X. Zhu, Org. Biomol. Chem. 2016 , 14, 6577-6590.

40. W. Zhang, D. Wei, M.-S. Tang, J. Org. Chem. 2013, 78 , 11849-11859.

41. Q.-C. Zhang, X. Li, X. Wang, S.-J. Li, L.-B. Qu, Y. Lan, D. Wei, Org. Chem. Front.2019 , 6 , 679-687.

42. X. Li, M. Tang, Y. Wang, Y. Wang, Z. Li, L.-B. Qu, D. Wei, Chem. Asian J. 2018 , 13 , 1710-1718.

43. X. L. Huang, X. Y. Chen, S. Ye,J. Org. Chem. 2009 , 74, 7585-7587.

44. H. Lv, X. Y. Chen, L. H. Sun, S. Ye, J. Org. Chem. 2010, 75 , 6973-6976.

45. T. Wang, X. L. Huang, S. Ye,Org. Biomol. Chem. 2010 , 8 , 5007-5011.

46. X. N. Wang, P. L. Shao, H. Lv, S. Ye, Org. Lett. 2009, 11 , 4029.

47. Y. R. Zhang, L. He, X. Wu, P. L. Shao, S. Ye, Org. Lett. 2008, 10 , 277.

48. Q. Liu, L. Sun, S.-J. Li, X. Li, L.-B. Qu, Y. Lan, D. Wei, Chem. Asian J. 2019 ,14, 2000-2007.

49. X. Wei, R. Fang, L. Yang, Catal. Sci. Technol. 2015 , 5 , 3352-3362.

50. P.-C. Tu, L. Zhou, A. M. Kirillov, R. Fang, L. Yang, Org. Chem. Front. 2018 ,5 , 1356-1365.

51. P. Verma, P. A. Patni, R. B. Sunoj, J. Org. Chem. 2011 , 76 , 5606-5613.

52. W. Zhang, Y. Zhu, D. Wei, Y. Li, M. Tang, J. Org. Chem. 2012, 777, 10729-10737.

53. H. Zhang, H. Xu, H. Bai, D. Wei, Y. Zhu, W. Zhang, Org. Chem. Front. 2018 , 5 , 1493-1501.

54. S. J. Ryan, L. Candish, D. W. Lupton, Chem. Soc. Rev. 2013 , 42 , 4906-4917.

55. S. D. Sarkar, A. Biswas, R. C. Samanta, A. Studer, Chem. Eur. J. 2013 , 19 , 4464-4678.

56. H. U. Vora, P. Wheeler, T. Rovis, Adv. Synth. Catal. 2012 , 354, 1617-1639.

57. J. Mahatthananchai, J. W. Bode,Acc. Chem. Res. 2014, 47 , 696-707.

58. L. R. Shapiro, S. Samuels, L. Breslow, T. Camacho, Am. J. Public Health 1983 ,73 , 773-778.

59. M. Tumer, M. Aslantas, E. S,ahin, N. Deligon, Spectrochim. Acta, Part A 2008, 70 , 477-481.

60. A. Patra, A. James, T. K. Das, A. T. Biju, J. Org. Chem. 2018 , 83 , 14820-14826.

61. Y. Xia, Y. Liang, Y. Chen, M. Wang, L. Jiao, F. Huang, S. Liu, Y. Li, Z.-X. Yu, J. Am. Chem. Soc. $2007,129,3470-3471$.

62. W. J. Hehre, R. Ditchfield, J. A. Pople, J. Chem. Phys. 1972, 56 , 2257-2261.

63. M. J. Frisch, G. W. Trucks, H. B. Schlegel, G. E. Scuseria, M. A. Robb, J. R. Cheeseman, G. Scalmani, V. Barone, B. Mennucci, G. A. Petersson, H. Nakatsuji, M. Caricato, X. Li, H. P. Hratchian, A. F. Izmaylov, J. Bloino, G. Zheng, J. L. Sonnenberg, M. Hada, M. Ehara, K. Toyota, R. Fukuda, J. Hasegawa, M. Ishida, T. Nakajima, Y. Honda, O. Kitao, H. Nakai, T. Vreven, J. A. Montgomery, J. E. P. Jr., F. Ogliaro, M. Bearpark, J. J. Heyd, E. Brothers, K. N. Kudin, V. N. Staroverov, T. Keith, R. Kobayashi, J. Normand, K. Raghavachari, A. Rendell, J. C. Burant, S. S. Iyengar, J. Tomasi, M. Cossi, N. Rega, J. M. Millam, M. Klene, J. E. Knox, J. B. Cross, V. Bakken, C. Adamo, J. Jaramillo, R. Gomperts, R. E. Stratmann, O. Yazyev, A. J. Austin, R. Cammi, C. Pomelli, J. W. Ochterski, R. L. Martin, K. Morokuma, V. G. Zakrzewski, G. A. Voth, P. Salvador, J. J. Dannenberg, S. Dapprich, A. D. Daniels, O. Farkas, J. B. Foresman, J. V. Ortiz, J. Cioslowski, D. J. Fox. Gaussian, Inc.: Wallingford CT, 2010. 
64. Y. Zhao, D. G. Truhlar, Theor. Chem. Acc. 2008 , 120 , 215-241.

65. Y. Zhao, D. G. Truhlar, J. Chem. Theory Comput. 2008 , 4, 1849-1868.

66. Y. Zhao, D. G. Truhlar,Acc. Chem. Res. 2008 , 41 , 157-167.

67. W. Sang-Aroon, V. Ruangpornvisuti, Int. J. Quantum Chem 2007 , 108 , 1181-1188.

68. B. Mennucci, J. Tomasi, J. Chem. Phys. 1996 , 106 , 5151-5158.

69. C. Gonzalez, H. B. Schlegel,J. Phys. Chem. 1990 , 94 , 5523-5527.

70. C. Gonzalez, H. B. Schlegel,J. Chem. Phys. 1989 , 90 , 2154-2161.

71. R. Krishnan, J. S. Binkley, R. Seeger, J. A. Pople, J. Chern. Phys. 1980 , 72 , 650-654.

72. A. V. Marenich, C. J. Cramer, D. G. Truhlar, J. Phys. Chem. B 2009, 113 , 4538-4543.

73. A. V. Marenich, C. J. Cramer, D. G. Truhlar, J. Phys. Chem. B 2009 , 113 , 6378-6396. 